\title{
CT appearances, patterns of progression, and follow-up of COVID-19: evaluation on thin-section CT
}

\author{
Chun-Shuang Guan ${ }^{1 \dagger}$, Zhi-Bin Lv ${ }^{1 \dagger}$, Jing-Jing Li' ${ }^{1}$, Yan-Ni Du ${ }^{1}$, Hui Chen ${ }^{1}$, Tao Cui ${ }^{1}$, Ning Guo ${ }^{2}$, \\ Bu-Dong Chen ${ }^{1^{*}}$ and Ru-Ming Xie ${ }^{1^{*}}$
}

\begin{abstract}
Background: To retrospectively analyze CT appearances and progression pattern of COVID-19 during hospitalization, and analyze imaging findings of follow-up on thin-section CT.

Methods: CT findings of 69 patients with COVID-19 were evaluated on initial CT, peak CT, and pre-discharge CT. CT pattern were divided into four types on CT progression. Lesion percentage of pulmonary lobe (lobe score) was graded. Correlation analysis was made between scores and intervals. 53 patients were followed up by CT.

Results: Among 69 patients, 33.3\% exhibited improvement pattern, 65.2\% peak pattern, 1.5\% deterioration pattern, and $0 \%$ fluctuation pattern. The lobe scores were positively correlated with most of intervals. It was more common to observe consolidation, pleural thickening and pleural effusion on the peak $C T$, and irregular line and reticulation on pre-discharge CT. The peak-initial interval were shortened when the initial CT with consolidation and pleural thickening. The intervals were extended when the irregular lines appeared on peak CT and reticulation on pre-discharge CT. Among 53 follow-up patients, 37.7\% showed normal chest CT, and 62.3\% showed viral pneumonia remained that mainly included GGO (100.0\%) and irregular lines (33.3\%).

Conclusions: COVID-19 displayed different appearances on CT as progressing. The peak pattern was the most common progression pattern. The CT appearances showed closely related to the intervals. The COVID-19 pneumonia can be remained or completely absorbed on CT with follow-up.
\end{abstract}

Keywords: COVID-19, Pneumonia, Infectious disease medicine, Multidetector computed tomography

\section{Key points}

- The most progression pattern was peak pattern.

- Lobe score showed a positive correlation with intervals

- Intervals were more extended as irregular lines and reticulation on $\mathrm{CT}$.

\footnotetext{
*Correspondence: chenbudong00@126.com; mingrux1@163.com

${ }^{\dagger}$ Chun-Shuang Guan and Zhi-Bin Lv contributed equally to this work and were considered co-first authors

${ }^{1}$ Department of Radiology, Beijing Ditan Hospital, Capital Medical University, No. 8 Jingshun East Street, Chaoyang District, Beijing, China

Full list of author information is available at the end of the article
}

- COVID-19 can be remained or completely absorbed on chest $\mathrm{CT}$ with follow-up.

\section{Background}

Coronavirus disease 2019 (COVID-19), which is caused by severe acute respiratory syndrome-associated coronavirus 2 (SARS-CoV-2). COVID-19 is first reported in Wuhan, China on December 31, 2019 [1], which spreads and progresses very quickly. By March 11 2020, the World Health Organization (WHO) classified the outbreak as a pandemic [2]. COVID-19 is still diffused across different countries at present. As of April 10, 2021, there were 
133,552,774 confirmed cases and 2,894,295 deaths worldwide [3]. At present, the United States is the country with the largest number of confirmed patients $(30,615,849$ confirmed cases, and 553,801 deaths), following by Brazil (13,193,205 confirmed cases, and 340,776 deaths) and India (13,060,542 confirmed cases, and 167,642 deaths) [4]. Computed tomography (CT) scanning was one of the important examinations for a diagnosis of COVID-19 $[5,6]$, and the CT images change rapidly in COVID-19. The patients show different findings of chest CT in different periods, [7-10]. Ground-glass opacity (GGO) is presented as the main manifestation in the early stage, crazy-paving pattern mainly in the progressive stage, consolidation mainly in the peak stage, the consolidation is gradually absorbed and fibrotic changes arise in the recovery stage [7-10]. To obtain better understanding of COVID-19 progression and follow-up changes on CT, we carried out the retrospective study on the CT appearances, pattern of progression and follow-up of discharged patients.

\section{Methods \\ Materials}

This study was approved by the Institutional Review Committee and the Ethics Committee of the institution (Beijing Ethics 2020 No. 022-01). Written informed consent for the retrospective analysis study was waived by the Institutional Review Board. All patients who were diagnosed as having SARS-CoV-2 by gene sequencing by fluorescence-based real-time reverse transcriptionpolymerase chain reaction (RT-PCR) were collected from January 12 to May 31, 2020. The inclusion criteria were: (1) adult patients ( $\geq 18$ years old); (2) patient with positive findings of COVID-19 pneumonia on chest thin-section CT; (3) patients discharged from hospital, who met the discharge criteria, including the body temperature returning to normal for more than 3 days, the respiratory symptoms obviously improving, the lung imaging showing the acute exudative lesions obviously improving, and the nucleic acid test of respiratory tract samples such as sputum and nasopharyngeal swabs for two consecutive times was negative (the sampling time interval was at least $24 \mathrm{~h}$ ). The exclusion criteria were: (1) patients without chest CT scans; (2) patients with other diffuse pulmonary disease in previous medical history or patients with other pathogens infection of clinical diagnosis; (3) patients without COVID-19 pneumonia on chest CT, that was, COVID-19 patients showed the normal chest CT; (4) patients remaining in hospital; (5) patients who did not return to the hospital for checks when collecting follow-up data. The clinical features (fever, cough, expectoration, chest tightness, dyspnea, runny nose, fatigue, hypogeusia, anosmia, diarrhea) and previous medical history were collected.

\section{Scanning equipment and scanning method}

All patients underwent 16-slice spiral CT (Siemens, Sensation CT, Forchheim, Germany). All patients were supine with arms raised and held their breath at the end of inhalation during scanning. The scanning range was from the tip to the base of the lung. The slice thickness was $5.0 \mathrm{~mm}$, and then the thin-section images with a slice thickness of $1.5 \mathrm{~mm}$ were reconstructed by a lung reconstruction algorithm. The tube voltage was $130 \mathrm{kV}$. The tube current was tube current modulation. The matrix was $512 \times 512$.

\section{Qualitative evaluation on chest CT}

Two chest radiologists with 13 and 15 years of working experience independently evaluated the images on the Picture Archiving and Communication System (Carestream Health, Rochester, NY, US) and reached agreement through consensus. Initial CT was defined as first CT examination after the hospital visit. Peak CT was that with the largest size of lesion on CT or the highest attenuation of the density when no change in the size during COVID-19 progression. Pre-discharge CT was defined as the last CT examination before patients discharged from hospital. The time intervals included initial interval (interval between onset of symptoms and initial CT examination), peak interval (interval between onset of symptoms and peak CT examination), pre-discharge interval (interval between onset of symptoms and predischarge CT examination), peak-initial interval (interval between peak $\mathrm{CT}$ and initial $\mathrm{CT}$ examination), pre-discharge-peak interval (interval between pre-discharge CT and peak CT examination) (Fig. 1).

Based on CT progression during hospitalization, the CT patterns were divided into four types. Type I (improvement pattern) exhibited improving on CT. Type II (peak pattern) showed initial CT deteriorating to a peak level following by improvement. Type III (deterioration pattern) was defined as deteriorating on CT. Type IV (fluctuation pattern) showed fluctuation changes with at least two peaks on CT.

The lesion distributions on $\mathrm{CT}$ were divided into subpleural distribution and peribronchovascular bundle distribution. Subpleural area was defined as mainly distributing along the subpleural $1 / 3$ region. Peribronchovascular bundle distribution was defined as mainly distributing around bronchovascular bundle.

CT appearances included GGO, consolidation, crazypaving pattern, air bronchogram, irregular line, reticulation, pleural thickening, pleural effusion, nodule and lymphadenopathy. GGO referred to a fuzzy increase 


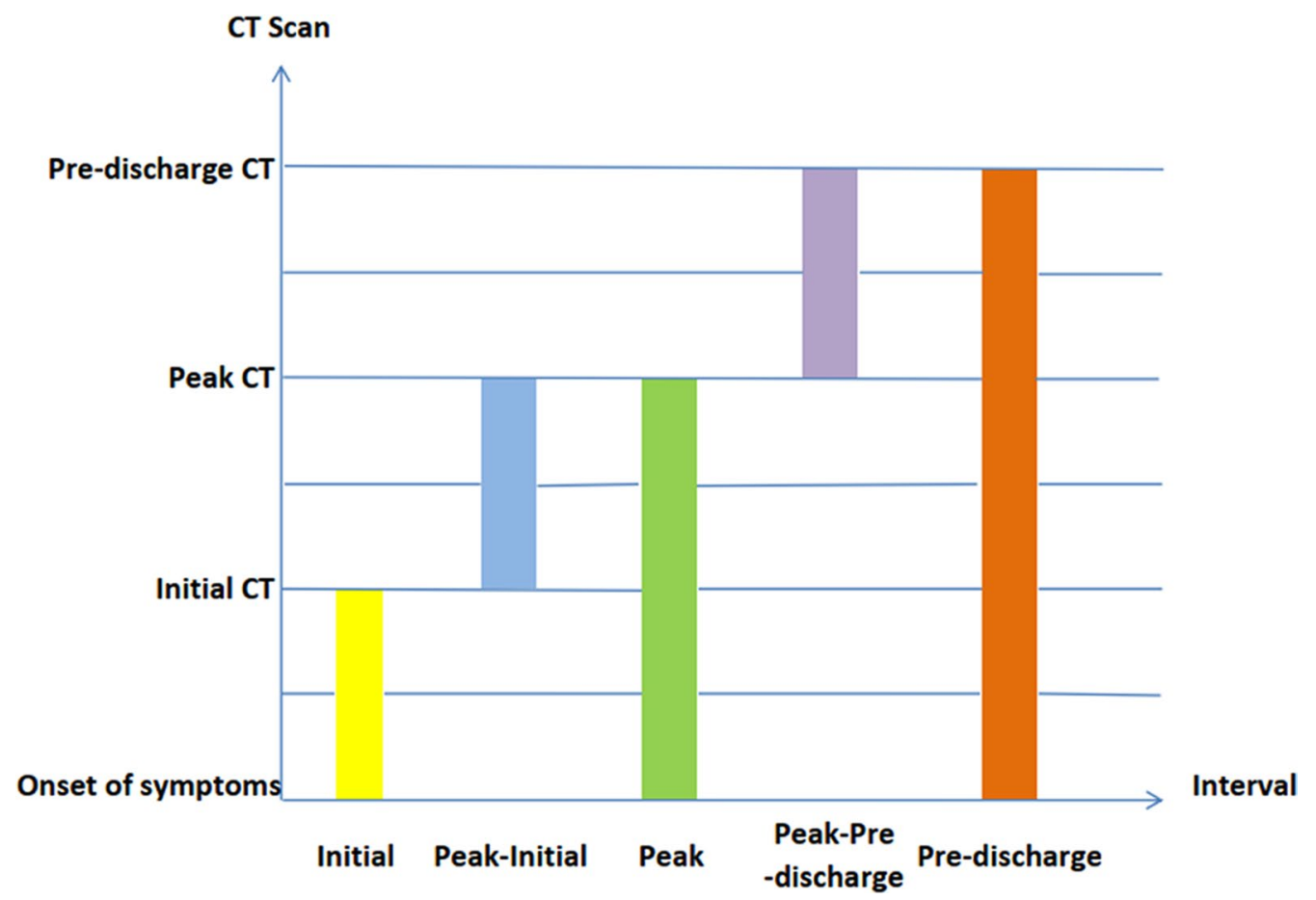

Fig. 1 Schematic diagram of different time intervals

in lung density that does not cover blood vessels. Consolidation was defined as increased attenuation in density that covered blood vessels. Crazy-paving pattern referred to reticular shadow superimposed with GGO. Air bronchogram was defined as bronchus containing gas outlined by high attenuation of lung parenchyma. Irregular line referred to irregular linear shadow with thickness of $1-3 \mathrm{~mm}$, excluding interlobular septa thickening and intralobular lines. Reticulation was formed only by interlaced lines without GGO. Nodule referred to round shadows of high attenuation with clear or fuzzy edges, $<3.0 \mathrm{~cm}$ in diameter. Pleural effusion refers to abnormal fluid in pleural cavity. Lymphadenopathy was defined as lymph nodes with $>1.0 \mathrm{~cm}$ in short diameter [11].

\section{Quantitative evaluation on chest CT}

Pulmonary lesions were scored according to the lesion size. The scoring criterion was defined as the percentage of lesions in one lobe (here termed lobe score). The lobes included upper, middle and lower lobes of right lung, and apicoposterior and anterior segments of left upper lobe, the upper and lower lingula segments of left upper lobe, and lower lobe of left lung. The lobe scores were as follows: 0 with no lesion in the lung, 1 with $0 \%<$ percentage $(P)<25 \%$, score of 2 with $25 \% \leq P<50 \%$, score of 3 with $50 \% \leq P<75 \%$, score of 4 with $P \geq 75 \%$. The total score of the whole lung was 1-24.

\section{Follow-up CT}

With follow-up, when pneumonia lesions were completely absorbed on $\mathrm{CT}$, the $\mathrm{CT}$ was taken as the followup CT. If there were pneumonia lesions remained on $\mathrm{CT}$ all the time, the last CT was taken as the follow-up CT. And the follow-up patients were divided into two groups, including absorption group and residual group. The absorption group referred to the complete absorption of viral pneumonia lesions on $\mathrm{CT}$, and the residual group refers to the viral pneumonia lesions remaining on CT.

\section{Statistical analysis}

Statistical analysis was performed using SPSS17.0 software (IBM, Armonk, NY, US). Continuous data were expressed as the mean and standard deviation or median (25th percentile, 75th percentile), and categorical data were expressed as the frequency. The distributions and imaging manifestations on initial $\mathrm{CT}$, peak $\mathrm{CT}$, and pre-discharge CT were analyzed by Chi-square test or Fisher's extract test. Analysis of repeated measures of variance and Friedman analysis were applied to the lobe scores on CT. Correlations were analyzed by multivariate linear regression. Absolute values of correlation coefficients $(r)$ were graded as: $0.15-0.24$, very low; $0.25-0.49$, low; $0.50-0.69$, moderate; $0.70-0.89$, high; $0.90-1.00$, very high. The analysis of variance and Mann-Whitney test were used to analyze the statistical differences of 
intervals on the imaging manifestations of initial, peak, and pre-discharge CTs. The follow-up CTs were analyzed by t test, Chi-square test, or Fisher's extract test. $p$ value < 0.05 was considered statistically difference.

\section{Results}

\section{Basic information of patients}

This study collected 135 patients with COVID-19. Sixty-six patients were excluded. Sixty-nine patients were enrolled and analyzed retrospectively (37 females and 32 males, mean age $46.058 \pm 15.641$ years; range, $18-80$ years). The 67 patients were evaluated on initial CTs because the two of 69 patients presented as the normal CT but showed the COVID-19 pneumonia on the peak CT and pre-discharge CT. All of the 69 patients were evaluated on the peak $\mathrm{CT}$ and pre-discharge CT. The most common clinical features were fever $(76.8 \%, 53 / 69)$, following cough $(68.1 \%, 47 / 69)$, dry throat $(29.0 \%, 20 / 69)$, runny nose $(27.5 \%, 19 / 69)$, fatigue $(27.5 \%, 19 / 69)$, headache $(24.6 \%, 17 / 69)$, expectoration $(21.7 \%, 15 / 69)$, chest tightness $(13.0 \%, 9 / 69)$, diarrhea $(11.6 \%, 8 / 69)$ dyspnea $(7.2 \%, 5 / 69)$, anosmia $(8.7 \%, 6 / 69)$, hypogeusia $(4.35 \%, 3 / 69)$. Some of the patients were accompanied by previous medical history, including hypertension $(14.5 \%, 10 / 69)$, heart disease $(5.8 \%, 4 / 69)$, diabetes mellitus $(7.2 \%, 5 / 69)$, and cerebrovascular disease $(2.9 \%, 2 / 69)$. The CT patterns of the 69 patients on CT showed that 23 (33.3\%) were type I, 45 (65.2\%) type II, one (1.5\%) in Type III, and none Type IV.
The intervals of the 69 patients were as follows: initial interval (5.81 \pm 3.548 days), peak interval ( $9.80 \pm 3.763$ days), pre-discharge interval (20.97 \pm 7.456 days), peak-initial interval $(3.99 \pm 3.281$ days), pre-discharge-peak interval $(11.19 \pm 6.330$ days). The peak-initial interval was significantly shorter than pre-discharge-peak interval $(p<0.001)$ (Fig. 2).

\section{Distribution}

All 69 cases showed mainly distributing along the subpleural area on the initial, peak, and pre-discharge CTs, and nearly $1 / 3$ of cases accompanying by distribution around peribronchivascular bundle (Table 1). The proportion of involvement in the lower lobes was slightly higher than that in the upper and middle lobes $(p=0.453-0.947) \quad$ (Table 1) (Figs. 3, 4, 5).symptoms shows the round ground-glass

\section{Correlation between scores and intervals}

The lobe score on peak CT $(7.17 \pm 4.762)$ was markedly higher than that of initial CT $(5.55 \pm 3.974, p<0.001)$ and pre-discharge CT $(5.67 \pm 3.563, p<0.001)$ (Fig. 6).

Lobe scores showed positive correlations with the intervals $(r$ range, $0.301-0.669, p<0.001)$ except the peakinitial interval $(p=0.210-0.400)$ (Table 2).

\section{CT appearances}

All 69 patients displayed GGO on the initial, peak, and pre-discharge CTs. Round GGOs on the initial CT

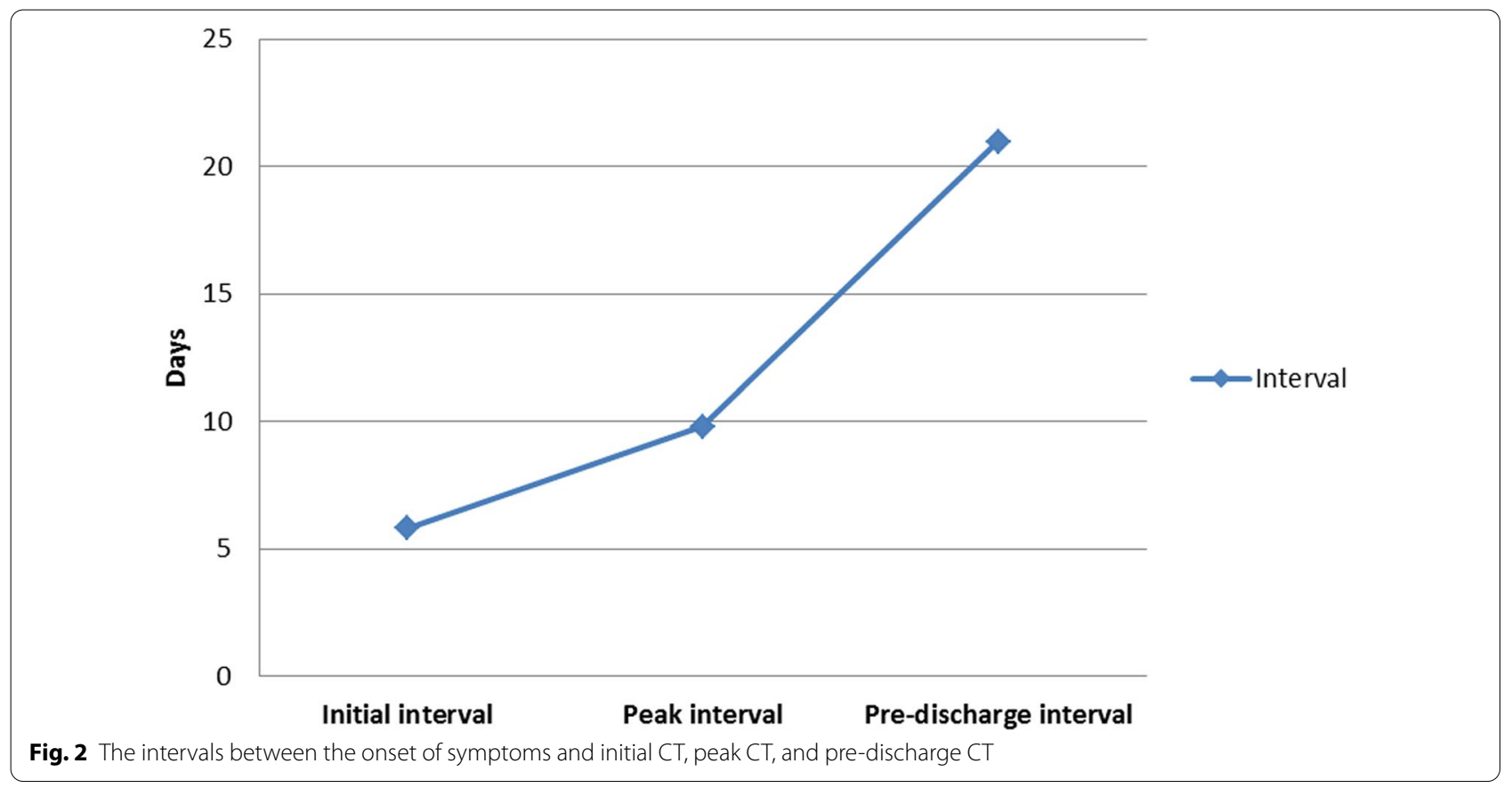


Table 1 Distribution of initial, peak, and pre-discharge CTs of COVID-19 during hospitalization

\begin{tabular}{|c|c|c|c|c|}
\hline & Initial CT (\%) & Peak CT (\%) & $\begin{array}{l}\text { Pre- } \\
\text { discharge } \\
\text { CT (\%) }\end{array}$ & $p$ value \\
\hline No & $67^{a}$ & 69 & 69 & \\
\hline $\begin{array}{l}\text { Subpleural distri- } \\
\text { bution }\end{array}$ & $45(67.2)$ & $47(68.1)$ & $46(66.7)$ & 0.983 \\
\hline $\begin{array}{l}\text { Mixed } \\
\quad \text { distribution }\end{array}$ & $22(32.8)$ & $22(31.9)$ & $23(33.3)$ & \\
\hline \multicolumn{5}{|l|}{ Right lung } \\
\hline Upper lobe & $44(65.7)$ & $50(72.5)$ & 47 (68.1) & 0.687 \\
\hline Middle lobe & $38(56.7)$ & $43(62.3$ & $41(59.4)$ & 0.801 \\
\hline Lower lobe & $57(85.1)$ & $60(87.0)$ & $59(85.5)$ & 0.947 \\
\hline \multicolumn{5}{|l|}{ Left lung } \\
\hline Upper lobe & $45(67.2)$ & $53(76.8)$ & $49(71.7)$ & 0.453 \\
\hline Lower lobe & $56(83.6)$ & $61(88.4)$ & $60(87.0)$ & 0.703 \\
\hline
\end{tabular}

${ }^{\text {a }}$ Two of 69 patients show no COVD-19 on CT

${ }^{\mathrm{b}}$ Mixed distribution $=$ distribution of subpleural and bronchovascular bandle
(53.7\%) more commonly observed than that on both of peak CT $(31.9 \%, p=0.010)$ and pre-discharge CT $(31.9 \%$, $p=0.010$ ) (Table 3) (Figs. 3, 7).

Consolidations on peak CT $(94.2 \%)$ were more common than those on initial CT $(73.1 \%, p=0.006)$ and predischarge CT $(65.2 \%, p<0.001)$ (Figs. 3, 4, 5, 7). 49.3\% of the pre-discharge CT displayed the crazy-paving pattern, significantly fewer than the initial CT $(79.1 \%, p<0.001)$ and peak CTs $(85.5 \%, p<0.001)$. Air bronchograms (59.4\%) were less commonly found on pre-discharge CT than on initial $(89.6 \%, p<0.001)$ and peak CTs $(92.8 \%$, $p<0.001)$. Irregular lines on initial CT $(46.3 \%)$ were less evident than those on peak $(68.1 \%, p=0.010)$ and predischarge CTs $(73.9 \%, p=0.001)$. Reticulations appeared on $23.2 \%$ of pre-discharge CT, more common than that on peak CT $(1.4 \%, p<0.001)$, and absent on initial CT (Figs. 3, 5, 7). Pleural thickenings were more commonly found on peak CT $(97.1 \%)$ than initial CT $(77.6 \%$, $p=0.001)$ and pre-discharge CT $(78.3 \%, p=0.001)$ (Figs. 3, 4, 5). The pleural effusions were more commonly seen on peak CT (10.1\%) than on initial CT $(4.5 \%$,
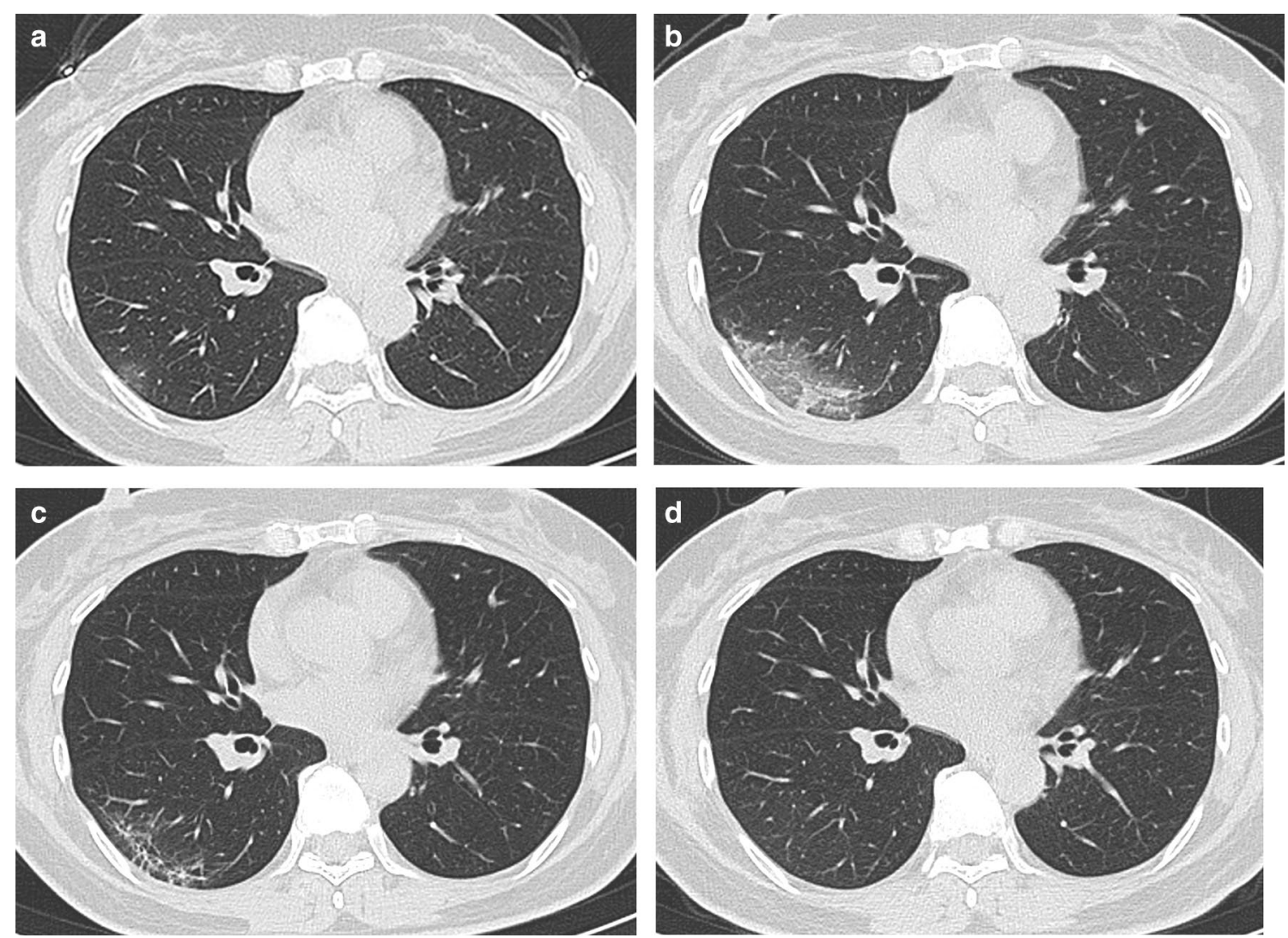

Fig. 3 Female, 50-year-old, COVID-19. a The initial CT obtained 4 days from onset of symptoms shows the round ground-glass opacity around the subpleural area of the right lower lobe. b The peak CT obtained 6 days later shows the large patchy ground-glass opacity and a few of consolidation around the subpleural area of the right lower lobe. c The pre-discharge CT obtained 14 days later shows reticulation, irregular lines, and pleura thickening. d. Axial CT. The follow-up CT obtained 64 days later shows a normal chest CT 

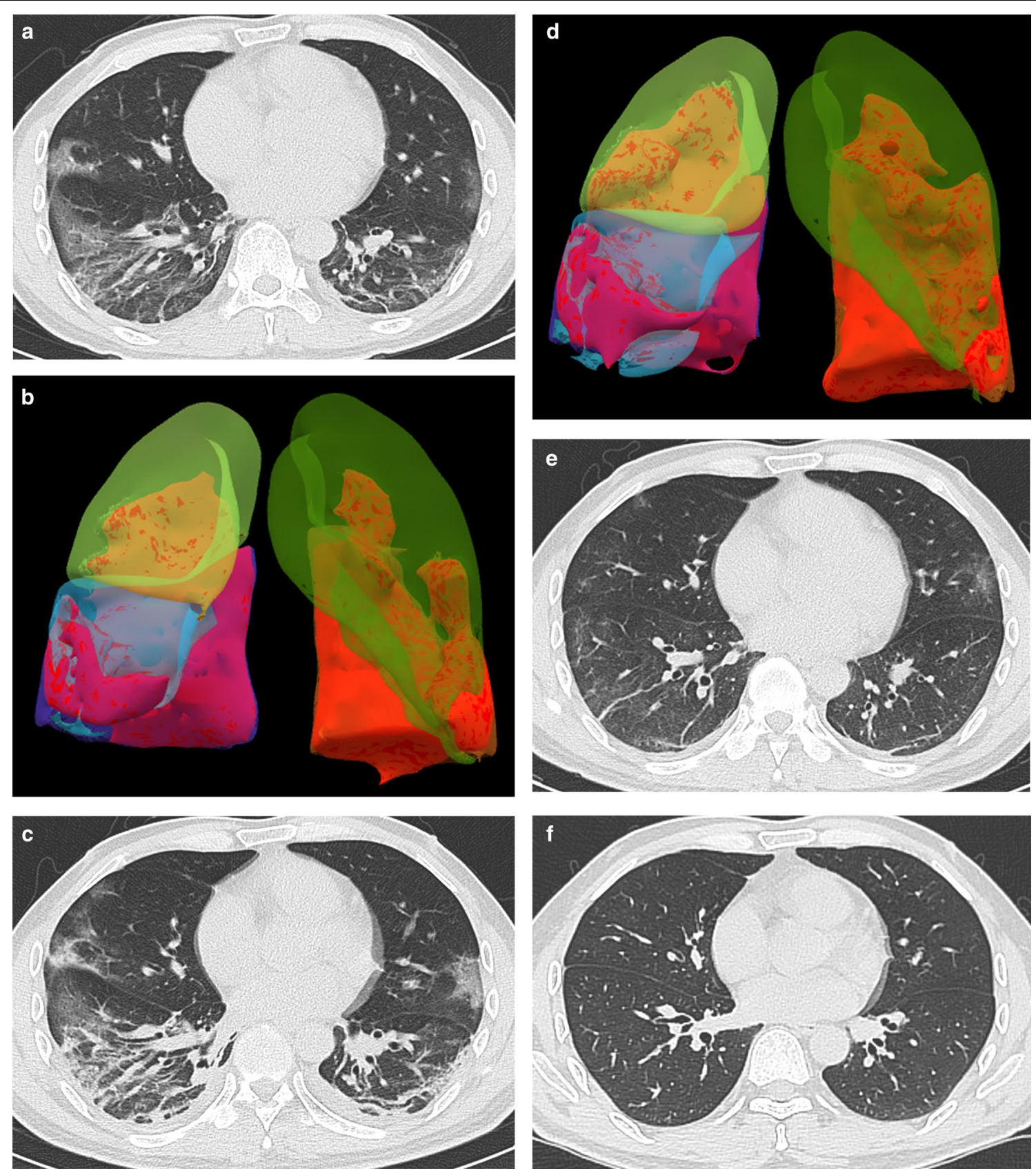

Fig. 4 Male, 45-year-old, COVID-19. a Axial CT and (b) Volume Render (VR) Imaging. The initial CT and VR imaging obtained 7 days from onset of symptoms. The CT shows the patchy ground-glass opacity, crazy-paving pattern, irregular lines, and consolidation around the subpleural area, and pleura thickening. The VR imaging shows the lesions involve the bilateral lungs. c Axial CT and (d) VR Imaging. The peak CT and VR imaging obtained 3 days later. The CT shows that ground-glass opacity decrease, consolidation irregular lines, and pleural thickening increase, and bilateral pleura effusion occurred. The VR imaging shows the lesion size significantly increases. e Axial CT. The pre-discharge CT obtained 8 days later. The CT shows ground-glass opacity, irregular lines, and pleural thickening adhesions remain. $\mathbf{f}$ Axial CT. The follow-up CT obtained 34 days later shows a normal chest CT

$p=0.349)$, and absent on pre-discharge CT $(p=0.020)$ (Table 3) (Figs. 4, 7).

Other imaging findings included nodules in seven cases, but none lymphadenopathy.

\section{Comparing imaging manifestations of intervals}

There were different manifestations in the initial CT, peak CT, and pre-discharge CT (Fig. 7). When consolidations, air bronchograms, and irregular lines were 

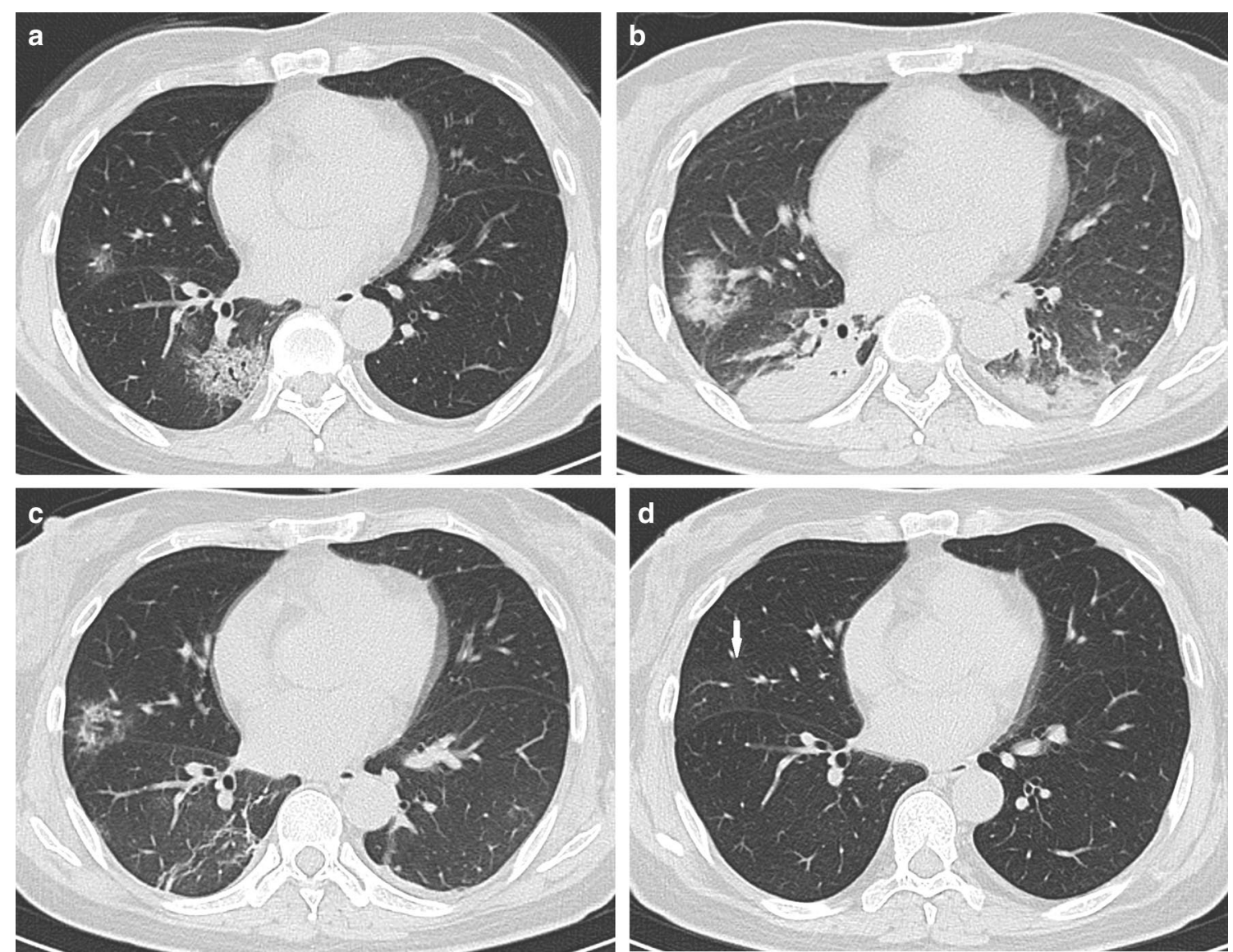

Fig. 5 Female, 55-year-old, COVID-19. a The initial CT obtained 5 days from onset of symptoms shows the round ground-glass opacity (arrow) around the subplerual area of the left lower lobe. $\mathbf{b}$ The peak CT obtained 9 days later shows the large patchy consolidations and pleural thickening increase much more and air bronchograms are observed. c The pre-discharge CT obtained 8 days later shows consolidation, ground-glass opacity, irregular lines, reticulation, and pleura thickening. $\mathbf{d}$ The follow-up CT obtained 45 days later shows a few of ground-glass opacity remained in right middle lobe (arrow)

found on the initial $\mathrm{CT}$ and irregular lines on the predischarge $\mathrm{CT}$, the initial intervals were much more extended than no these findings on the initial and predischarge CTs. When irregular lines were observed on the peak $\mathrm{CT}$ and irregular lines and air bronchograms on the pre-discharge $\mathrm{CT}$, the peak intervals were more extended (Table 4). The pre-discharge intervals were more extended when irregular lines were found on peak $\mathrm{CT}$ and reticulations on pre-discharge $\mathrm{CT}$, while the pre-discharge intervals were more shortened as consolidation and crazy-paving pattern on pre-discharge $\mathrm{CT}$ (Table 4).

The peak-initial intervals were more shortened when consolidations and pleural thickenings on the initial CT (Fig. 4). The pre-discharge-peak intervals were more extended as crazy-paving pattern on the initial CT, irregular lines on the peak $\mathrm{CT}$, and reticulations on the pre-discharge $\mathrm{CT}$, but shortened as consolidation and crazy-paving pattern on pre-discharge CT (Table 4).

\section{Follow-up}

Among 69 patients, 16 patients (23.2\%) did not come to hospital for checks, and the other 53 patients (76.8\%) were followed up. The 53 patients included 22 females and 31 males, and mean age $49.509 \pm 14.780$ years (range, 19-80 years). Median follow-up intervals were 60.0 (45.0, 89.5) days (range, 30-329 days). And there were 20 patients $(37.7 \%$ ) in absorption group ( 8 females and 12 males, and mean age $43.050 \pm 11.450$ years; range, 29-76 years; median follow-up interval, 68.0 (46.5, 109.8) days ) (Figs. 3, 4) and 33 patients (62.3\%) in residual group (14 females and 19 males, and mean age $53.424 \pm 15.337$ years; range, $19-80$ years; median follow-up interval, $56.0(44.5,83.0)$ days) (Fig. 5), respectively. Between the two groups, the gender and follow-up interval had no significantly statistical difference, but the age of the residual group was elder than that of the absorption group $(p=0.012)$. 


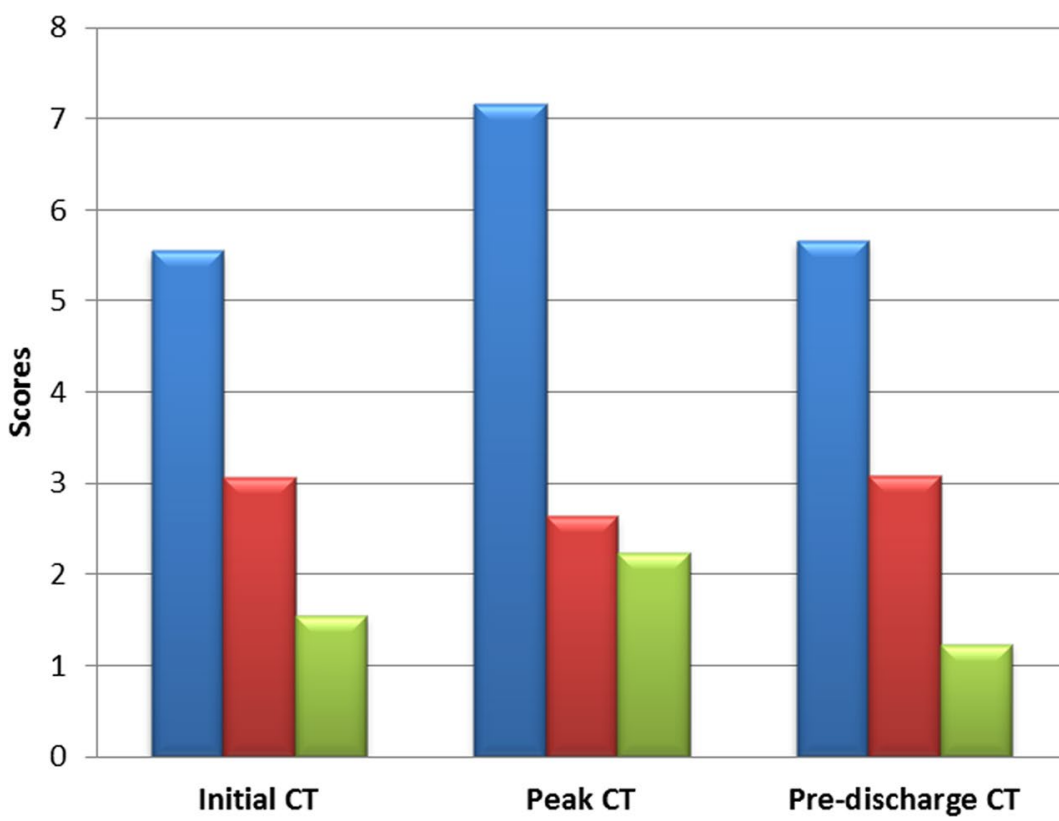

Fig. 6 The lobe score, ground-glass opacity score, and consolidation score on initial CT, peak CT, and pre-discharge CT

Table 2 Correlation coefficient between intervals and score of lobes percentage on initial, peak, and pre-discharge CTs of COVID-19 during hospitalization

\begin{tabular}{lcll}
\hline & Initial CT $(\boldsymbol{p}$ value $)$ & Peak CT $(\boldsymbol{p}$ value $)$ & Pre-discharge CT $(\boldsymbol{p}$ value $)$ \\
\hline Initial interval & $0.621(<0.001)$ & $0.417(<0.001)$ & $0.419(<0.001)$ \\
Peak interval & $0.465(<0.001)$ & $0.530(<0.001)$ & $0.488(<0.001))$ \\
Pre-discharge interval & $0.503(<0.001)$ & $0.669(<0.001)$ & $0.496(<0.001)$ \\
Peak-Initial interval & $-0.108(0.372)$ & $0.153(0.210)$ & $0.103(0.400)$ \\
Pre-discharge-peak interval & $0.320(0.008)$ & $0.482(<0.001)$ & $0.301(0.006)$ \\
\hline
\end{tabular}

Table 3 Comparison among imaging findings of initial, peak, and pre-discharge CTs of COVID-19 during hospitalization

\begin{tabular}{|c|c|c|c|c|}
\hline & Initial CT (\%) & Peak CT (\%) & Pre-discharge CT (\%) & $p$ value \\
\hline No & $67^{a}$ & 69 & 69 & \\
\hline Ground-glass opacity(GGO) & $67(100.0)$ & $69(100.0)$ & $69(100.0)$ & \\
\hline Round GGO & $36(53.7)$ & $22(31.9)$ & $22(31.9)$ & 0.011 \\
\hline Patchy GGO & $31(46.3)$ & $47(68.1)$ & $47(68.1)$ & \\
\hline Consolidation & $49(73.1)$ & $65(94.2)$ & $45(65.2)$ & $<0.001$ \\
\hline Crazy-paving pattern & $53(79.1)$ & $59(85.5)$ & $34(49.3)$ & $<0.001$ \\
\hline Air bronchogram & $60(89.6)$ & $64(92.8)$ & $41(59.4)$ & $<0.001$ \\
\hline Irregular lines & $31(46.3)$ & $47(68.1)$ & $51(73.9)$ & 0.002 \\
\hline Only reticulation & $0(0.0)$ & $1(1.4)$ & $16(23.2)$ & $<0.001$ \\
\hline Pleural thickening adhesions & $52(77.6)$ & $67(97.1)$ & $54(78.3)$ & 0.002 \\
\hline Pleural effusion & $3(4.5)$ & $7(10.1)$ & $0(0)$ & 0.015 \\
\hline
\end{tabular}

${ }^{\mathrm{a}}$ Two of 69 patients show no COVD-19 on CT 


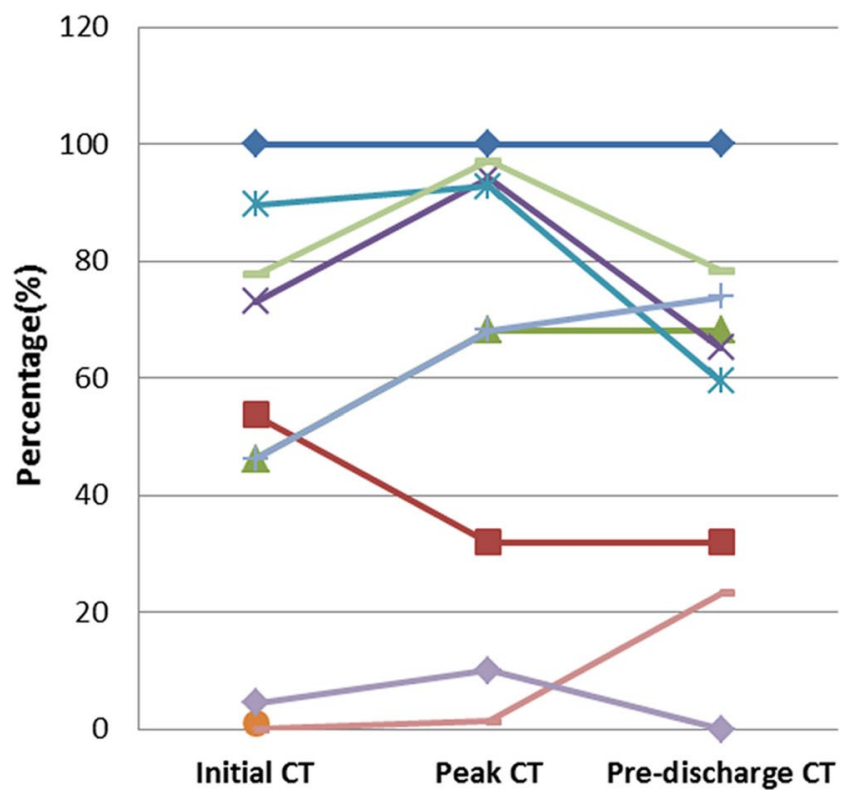

$\leadsto$ Ground-glass opacity

- Round Ground-glass opacity

-Patchy Ground-glass opacity

$\simeq$ Conlidation

Crazy-paving pattern

-Air bronchogram

_ Irregular lines

Reticulation

Pleural thickening adhesions

$\ldots$ Pleural effusion

Initial CT

Peak CT Pre-discharge CT

Fig. 7 The $C T$ appearences on the initial $C T$, peak $C T$, and pre-discharge $C T$

Table 4 Comparison of intervals on initial, peak, and pre-discharge CTs of COVID-19a during hospitalization

\begin{tabular}{|c|c|c|c|}
\hline & $\begin{array}{l}\text { Initial CT (yes }{ }^{b} \text {, days; no }{ }^{c} \text {, days; } p \\
\text { value) }\end{array}$ & $\begin{array}{l}\text { Peak } \mathrm{CT} \text { (yes }{ }^{\mathrm{b}} \text {, days; no }{ }^{\mathrm{c}} \text {, days; } p \\
\text { value) }\end{array}$ & $\begin{array}{l}\text { Pre-discharge } \mathrm{CT} \text { (yes }^{\mathrm{b}}, \text { days; } \text { no }^{\mathrm{c}} \\
\text { days; } p \text { value) }\end{array}$ \\
\hline Initial Interval & $\begin{array}{l}\text { Consolidation }(6.65 \pm 3.649 ; \\
3.78 \pm 1.957 ; 0.002) \\
\text { Air bronchogram }(6.23 \pm 3.524 ; \\
2.86 \pm 1.215 ; 0.015) \\
\text { Irregular line }(7.29 \pm 3.934 ; \\
\quad 4.67 \pm 2.586 ; 0.002)\end{array}$ & No significant statistically difference & $\begin{array}{l}\text { Irregular line }(6.31 \pm 3.603 ; 4.17 \pm 2.875 \\
0.026)\end{array}$ \\
\hline Peak Interval & No significant statistically difference & $\begin{array}{l}\text { Irregular line }(10.50 \pm 3.443 \\
\quad 8.17 \pm 3.892 ; 0.014)\end{array}$ & $\begin{array}{l}\text { Irregular line }(10.37 \pm 3.605 ; \\
\quad 7.89 \pm 3.579 ; 0.014) \\
\text { Air bronchogram }(10.54 \pm 3.641 \\
\quad 7.89 \pm 3.579 ; 0.014)\end{array}$ \\
\hline Pre-discharge Interval & No significant statistically difference & $\begin{array}{l}\text { Irregular line }(23.02 \pm 6.191 \\
\quad 17.13 \pm 8.374 ; 0.002)\end{array}$ & $\begin{array}{l}\text { Consolidation }(19.53 \pm 6.768 \\
23.92 \pm 8.032 ; 0.019) \\
\text { Crazy-paving pattern }(19.26 \pm 6.072 ; \\
22.80 \pm 8.345 ; 0.049) \\
\text { Reticulation }(25.13 \pm 7.356 ; \\
\quad 19.83 \pm 7.130 ; 0.012)\end{array}$ \\
\hline Peak-initial Interval & $\begin{array}{l}\text { Consolidation }(3.22 \pm 3.423 \\
5.78 \pm 2.211 ; 0.004) \\
\text { Pleural thickening }(3.40 \pm 3.339 \\
5.67 \pm 2.717 ; 0.019)\end{array}$ & No significant statistically difference & No significant statistically difference \\
\hline Pre-discharge-peak Interval & $\begin{array}{l}\text { Crazy-paving pattern }(12.26 \pm 6.048 ; \\
\quad 8.50 \pm 6.334 ; 0.044)\end{array}$ & $\begin{array}{l}\text { Irregular line }(12.54 \pm 5.995 \\
\quad 8.96 \pm 6.116 ; 0.023)\end{array}$ & $\begin{array}{l}\text { Consolidation }(9.80 \pm 5.814 \\
\quad 14.25 \pm 6.045 ; 0.004) \\
\text { Crazy-paving pattern }(9.44 \pm 5.177 \\
\quad 13.20 \pm 6.663 ; 0.011) \\
\text { Reticulation }(14.25 \pm 6.445 \\
\quad 10.47 \pm 5.947 ; 0.032)\end{array}$ \\
\hline
\end{tabular}

\footnotetext{
${ }^{a}$ The other imaging findings not shown in the table had no significantly statistically difference in intervals

${ }^{\mathrm{b}}$ Yes= imaging manifestation

${ }^{\mathrm{c}} \mathrm{No}=$ no imaging manifestation
} 
Residual lesions showed mainly distributing along the subpleural area on CT $(90.9 \%, 30 / 33)$, and only $9.1 \%$ (3/33) of cases distributing along subpleural area and around peribronchivascular bundle, which showed no significantly statistical difference with initial CT, peak CT, and pre-charge CT, respectively (Fig. 5).

GGO (100.0\%, 33/33) was still the most common CT pattern (Fig. 5), following irregular lines (33.3\%, 11/33), reticulation $(15.2 \%, 5 / 33)$, consolidation $(6.1 \%, 2 / 33)$ and pleural thickening adhesion (6.1\%, 2/33). Crazy-paving pattern, air bronchogram, and pleural effusion were not found. All the GGO presented as patchy GGO, not round GGO.

GGO morphology, consolidation, crazy-paving pattern, air bronchogram, and pleural thickening adhesions on follow-up CT showed significantly statistical difference with initial $\mathrm{CT}$, peak $\mathrm{CT}$, and pre-charge $\mathrm{CT}$, respectively $(p<0.001)$. Irregular lines $(33.3 \%)$ were less common than those on peak CT $(68.1 \%, p<0.001)$ and pre-discharge CT (73.9\%, $p=0.001)$, but not initial CT $(46.3 \%, p=0.218)$. Reticulations $(15.2 \%)$ were more common than those on initial CT $(0.0 \%, p=0.003)$ and peak CT $(1.4 \%, p=0.013)$, respectively, but not pre-charge CT $(23.2 \%, p=0.348)$. Pleural effusion $(0.0 \%)$ had no significantly statistical difference with initial CT (4.5\%, $p=0.549)$, peak CT $(10.1 \%, p=0.093)$, but pre-charge CT $(0.0 \%, p$ value was not applicable).

\section{Discussion}

COVID-19 showed the typical appearances of viral pneumonia on $\mathrm{CT}$, with rapid progress and obvious changes in a short time. Through our study, we found that, first, the most progression pattern was type II (65.2\%), and peak-initial interval was shorter than the pre-dischargepeak interval. Second, lobe score showed a positive correlation with intervals except peak-initial interval. Third, it was most commonly observed to round GGOs on initial CT, consolidations on peak $\mathrm{CT}$, and reticulations on pre-discharge CT (Table 3) (Figs. 3, 4, 5, 7). Fourth, the initial intervals were much more extended when consolidation, air bronchogram, and irregular lines on initial CT (Fig. 4). The intervals were more extended as irregular lines and reticulation on CT (Table 4). Fifth, the viral pneumonia can be remained or completely absorbed on CT with follow-up (Figs. 3, 4, 5).

So far this century, there have been three serious infectious diseases caused by coronavirus, one of which was Severe Acute Respiratory Syndrome (SARS) that broke out in 2003 in China and spread to the whole world [12]; the second time was Middle East Respiratory Syndrome (MERS) in Iran (in 2012) [13]; the third time was COVID-19, which was first reported to the WHO Country Office in China on 31 December, 2019, and became a global event $[1,2]$. COVID-19 can cause severe respiratory system Diseases. These infectious diseases caused by coronaviruses showed similar CT manifestations. Although part of their imaging findings superimposed, they also have their specific characteristics, especially in the dynamic changes of CT. Studies of SARS report that the peak pattern (70.3\%) is the most common type, following by the fluctuating (17.4\%), improving (7.3\%), and deteriorating patterns (5.1\%) [14]. In MERS cases, the most common type is the deteriorating pattern (53.3\%), following by the peak (40.0\%), fluctuating (6.7\%), and improving patterns $(0 \%)$ [15]. Here, we found that the most common type in our study was the peak pattern (65.2\%), similar to SARS. But the improving pattern (33.3\%) was significantly higher than that of SARS and MERS and the deteriorating pattern (1.5\%) was significantly lower than that of MERS and slightly lower than that of SARS. Fluctuating pattern were observed in $17.4 \%$ of SARS and $6.7 \%$ of MERS, but absent in our study. The peak pattern was the dominate type in SARS and COVID-19 while the deteriorating pattern was the most common type in MERS [14, 15]. The deteriorating pattern was the least common type, which might be due to all of our cases were from discharged patients. It was special to have none fluctuating pattern in this study, the reason of which was not clear. However, the two studies are based on chest radiographs that are overlay imaging, so some detail changes cannot be clearly found. Our study was based on CT that can exhibit much more clearly lesions in the lungs than chest radiographs. Therefore, our study could accurately present the progression of the disease than those two studies. In this study, the peakinitial interval $(7.65 \pm 6.203$ days) was much shorter than pre-discharge-peak interval (11.35 \pm 6.228 days) (Fig. 2). Accordingly, COVID-19 progressed rapidly to the peak period, and then took longer time to gradually resolve until discharge.

Lobe score of COVID-19 exhibited positively correlated to most of intervals but not to peak-initial interval. That was, the larger the lesion sizes were associated the longer intervals (Table 2). As the consolidation and pleural thickening on the initial CT emerged, the peak-initial interval shortened, the disease progressed more rapidly. The round GGO was characteristic on the initial CT (Table 4, Fig. 4). When the irregular lines appeared on peak $\mathrm{CT}$, indicating the occurrence of fibrotic changes, the peak, pre-discharge, and pre-discharge-peak intervals were extended. The intervals were also extended when the reticulations appeared on the CTs (Table 4). Therefore, when fibrosis manifestations (irregular lines and reticulation) occurred on CTs, intervals would be extended. 
The imaging manifestations are based on the pathology that has been report for SARS and MERS $[12,13,16$, 17]. Moreover, it has been proposed that the pathology of COVID-19 is similar to that of SARS and MERS [18, 19]. In the early stage, COVID-19 shows that the exudation and proliferation of acute lung injury manifesting as edema, infiltration of inflammatory cells, proliferation of Type II alveolar cells and fibroblasts, protein exudation, and vascular congestion [19]. In the progression stage, the pathology shows diffuse alveolar injury, exfoliated alveolar cells with fibromucous secretion, pulmonary edema, hyaline membrane formation, monocytes infiltration (mainly lymphocytes) in alveolar cell septa, marked protein and fibrin exudates in the lung parenchyma, diffuse thickening of the alveolar wall, and fibroblasts and Type II alveolar cell hyperplasia, while focal fibroblast embolism can be seen in the alveolar cavity, presenting as acute respiratory distress syndrome $[18,20]$. Then the most common manifestation of COVID-19 was GGO (Figs. 3, 4, 5, 7), which might be associated with alveolar edema, inflammatory cells infiltration, and thickening of the alveolar septum. Consolidation might be caused by alveolar edema, inflammatory cells, protein and cellulose-like mucus in the alveolar cavity. The crazy-paving pattern involved both parenchyma and interstitium. Both irregular lines and reticulation might represent the fibrotic changes, diffuse thickening of the alveolar wall, fibroblasts proliferation, thickening of alveolar septa, and the thickening intralobular lines. Pleural thickening and adhesion were also common imaging manifestations, which might be related to the infiltration of inflammatory cells in the alveolar septum, and fibroblasts [11, 18-20].

With follow-up in this study, the pulmonary lesions could be remained (62.3\%) or completely absorbed (37.7\%). And the ages of the patients in residual lesions group were elder than that of the patients in absorption group, which is in accord with the literature [21]. The most common CT pattern with follow-up was GGO, following irregular lines, and reticulation, which were similar to the literature [21, 22] (Fig. 5).

This study had some limitation. First, COVID-19 was an emerging infectious disease. The sample size was not large, but it could still indicate the imaging manifestations and dynamic changes of COVID-19. Second, the study mainly studied the dynamic changes of COVID-19 on chest $\mathrm{CT}$, as well as the correlation analysis between imaging performances and time interval. There were fewer data on clinical features in the present study. However, there are some studies on the correlation of clinical characteristics of COVID-19 in the literatures [21-23]. For example, GGO and consolidation are the main manifestations in the acute stage of the disease. With the progress of the disease, some patients show the lesions are completely absorbed, and some patients show residual fibrosis lesions. It has been reported in the literature that the diffusion function of patients is decreased in the acute stage, and the abnormal diffusion function will still appear when the residual fibrosis changes in the lung in the recovery stage [7-10, 21, 22, 24]. In the future research, we will focus on the correlation between clinical features and CT imaging changes. Third, whether the interstitial lesions could be completely absorbed or not needed more long-term follow-up observation.

\section{Conclusions}

In brief, COVID-19 can rapidly progress to the peak and gradually recovers. The most common pattern of progression was the peak pattern, following by improvement pattern and deterioration pattern. The intervals were closely related to lobe scores and CT appearances. The higher the lobe score, the longer the intervals. If consolidations, air bronchograms, and irregular lines on initial CT increase, the initial intervals will be shorter. If the irregular lines and reticulations appear on the peak $\mathrm{CT}$ and pre-discharge $\mathrm{CT}$, the intervals will be extended. After follow-up, COVID-19 pneumonia can be completely absorbed while GGO, irregular lines, and reticulation also can be remained.

\section{Abbreviations \\ COVID-19: Coronavirus disease 2019; CT: Computed tomography; GGO: Ground-glass opacity; Lobe Score: Percentage of lesions in one lobe; MERS: Middle east respiratory syndrome; RT-PCR: Fluorescence-based real-time reverse transcription-polymerase chain reaction; SARS: Severe acute respira- tory syndrome; SARS-CoV-2: Severe acute respiratory syndrome-associated coronavirus 2; WHO: World Health Organization.}

\section{Acknowledgements}

We are grateful to lain Charles Bruce from Zhejiang University School of Medicine for providing language editing services.

\section{Authors' contributions}

GCS and LZB analyzed and interpreted the patient data, and wrote the original draft. LJ and DYN collected data and carried out the statistical analysis. CH and $\mathrm{CT}$ analyzed the data and select images. GN performed the CT postprocessing technology. CBD and XRM designed the research and reviewed the manuscript. All authors read and approved the final manuscript.

\section{Funding}

The work was supported by Beijing Municipal Commission of Science and Technology (Grant Numbers Z201100005420012) and Health Science Promotion Project of Beijing (Grant Numbers 2020-TG-001).

\section{Availability of data and materials}

The datasets used and/or analysed during the current study are available from the corresponding author on reasonable request.

\section{Declarations}

Ethics approval and consent to participate

This study was approved by the Institutional Review Committee and the Ethics Committee of the institution (Beijing Ditan Hospital, Capital Medical 
University; Beijing Ethics 2020 No. 022-01). Written informed consent for the retrospective analysis study was waived by the Institutional Review Board.

\section{Consent for publication}

Written informed consent for the retrospective analysis study was waived by the Institutional Review Board (Beijing Ditan Hospital, Capital Medical University).

\section{Competing interests}

All financial and non-financial competing interests must be declared in this section.

\section{Author details}

1 Department of Radiology, Beijing Ditan Hospital, Capital Medical University, No. 8 Jingshun East Street, Chaoyang District, Beijing, China. ${ }^{2}$ Department of Clinical Research, Shukun (Beijing) Technology Co., Ltd., Jinhui Bd, Qiyang Rd, Chaoyang District, Beijing, China.

Received: 18 February 2021 Accepted: 20 May 2021

Published online: 10 June 2021

\section{References}

1. World Health Organization. https://www.who.int/emergencies/diseases/ novel-coronavirus-2019/interactive-timeline\#! Published 31 December 2020. Accessed 30 Mar 2021.

2. World Health Organization. https://www.who.int/zh/news-room/detail/ 08-04-2020-who-timeline---covid-19. Published 11 March 2020. Accessed 9 Apr 2020.

3. World Health Organization. https://www.who.int/emergencies/disea ses/novel-coronavirus-2019/situation-reports. Published 30 March 2020. Accessed 9 Apr 2021

4. World Health Organization. https://covid19.who.int/table. Published 30 March 2021. Accessed 30 Mar 2021.

5. National Health Commission of the Peple's Republic of China. Consensus on guidelines for the publication of the seventh trail version of the diagnosis and treatment plan of the novel coronavirus coronavirus. http:// www.nhc.gov.cn/yzygj/s7653p/202003/46c9294a7dfe4cef80dc7f591 2eb1989.shtml. Published 4 March 2020. Accessed 4 Mar 2020.

6. Bollineni VR, Nieboer KH, Döring S et al (2021) The role of CT imaging for management of COVID-19 in epidemic area: early experience from a University Hospital. Insights Imaging 12:10

7. Pan F, Ye T, Sun P et al (2020) Time course of lung changes at chest CT during recovery from coronavirus disease 2019 (COVID-19). Radiology 295:715-721

8. Shi H, Han X, Jiang N et al (2020) Radiological findings from 81 patients with COVID-19 pneumonia in Wuhan, China: a descriptive study. Lancet Infect Dis 20:425-434

9. Li M, Lei P, Zeng B et al (2020) Coronavirus disease (COVID-19): spectrum of CT findings and temporal progression of the disease. Acad Radiol 27:603-608
10. Zhao W, Zhong Z, Xie X et al (2020) Relation between chest CT findings and clinical conditions of coronavirus disease (COVID-19) pneumonia: a multicenter study. AJR Am J Roentgenol 214:1072-1077

11. Hansell DM, Bankier AA, MacMahon H et al (2008) Fleischner society: glossary of terms for thoracic imaging. Radiology 246:697-722

12. Tsang KW, Ho PL, Ooi GC et al (2003) A cluster of cases of severe acute respiratory syndrome in Hong Kong. N Engl J Med 348:1977-1985

13. Alsaad KO, Hajeer AH, Al Balwi M et al (2018) Histopathology of Middle East respiratory syndrome coronovirus (MERS-CoV) infection-clinicopathological and ultrastructural study. Histopathology 72:516-524

14. Wong KT, Antonio GE, Hui DS et al (2003) Severe acute respiratory syndrome: radiographic appearances and pattern of progression in 138 patients. Radiology 228:401-406

15. Das KM, Lee EY, Enani MA et al (2015) CT correlation with outcomes in 15 patients with acute Middle East respiratory syndrome coronavirus. AJR Am J Roentgenol 204:736-742

16. Bradley BT, Bryan A (2019) Emerging respiratory infections: The infectious disease pathology of SARS, MERS, pandemic influenza, and Legionella. Semin Diagn Pathol 36:152-159

17. Walker DH (2016) Value of autopsy emphasized in the case report of a single patient with middle east respiratory syndrome. Am J Pathol 186:507-510

18. Xu Z, Shi L, Wang Y et al (2020) Pathological findings of COVID-19 associated with acute respiratory distress syndrome. Lancet Respir Med 8:420-422

19. Tian S, Hu W, Niu L et al (2020) Pulmonary pathology of early-phase 2019 novel coronavirus (COVID-19) pneumonia in two patients with lung cancer. J Thorac Oncol 15:700-704

20. Garton LM, Duval EJ, Stroberg E et al (2020) COVID-19 autopsies, Oklahoma, USA. Am J Clin Pathol 153:725-733

21. Ning KJ, Su DJ, Gu JF et al (2020) The follow-up chest CT imaging of 67 discharged patients with COVID-19 pneumonia. Radiol Practice 35:1235-1238

22. Huang C, Huang L, Wang Y et al (2021) 6-month consequences of COVID-19 in patients discharged from hospital: a cohort study. Lancet 397:220-232

23. Mungmungpuntipantip R, Wiwanitkit $V$ (2020) Clinical features and chest CT manifestations of coronavirus disease (COVID-19). AJR Am J Roentgenol 215:W13

24. van den Borst B, Peters JB, Brink M et al (2020) Comprehensive health assessment three months after recovery from acute COVID-19. Clin Infect Dis 21:ciaa1750

\section{Publisher's Note}

Springer Nature remains neutral with regard to jurisdictional claims in published maps and institutional affiliations.

\section{Submit your manuscript to a SpringerOpen ${ }^{\circ}$ journal and benefit from:}

- Convenient online submission

- Rigorous peer review

- Open access: articles freely available online

- High visibility within the field

- Retaining the copyright to your article

Submit your next manuscript at $\boldsymbol{\Delta}$ springeropen.com 